\title{
Lack of rebound during intermittent transdermal treatment with glyceryl trinitrate in patients with stable angina on background $\beta$ blocker
}

Diana R Holdright, Richard J Katz, Christine A Wright, Jane L Sparrow, Ann K Sullivan, A David Cunningham, Kim M Fox

Abstract

Objective-To assess whether intermittent transdermal treatment with glyceryl trinitrate causes clinically significant rebound in patients maintained on $\beta$ blockers for stable angina pectoris.

Design-Serial treadmill exercise testing in a double blind, randomised, placebo controlled cross over trial. Baseline exercise testing was performed at 0900 and 1100 at visit 1 . Transdermal glyceryl trinitrate patches releasing $15 \mathrm{mg} / 24 \mathrm{~h}$ were applied at 2200 the evening before visits 2 and 3 , and exercise testing was performed at 0900 the next morning. The patch was removed and replaced with either an identical patch or matching placebo and exercise tests were repeated two hours later. The alternative treatment was given at visit 3 .

Setting-Tertiary referral centre.

Patients-14 patients with stable angina pectoris maintained on $\beta$ blocker treatment alone.

Main outcome measures-Time to angina, $1 \mathrm{~mm}$ ST segment depression, and total time, together with heart rate, systolic blood pressure, and ratepressure product.

Results-Active treatment improved treadmill performance at 0900 and 1100 . Time to angina, time to $1 \mathrm{~mm}$ ST segment depression, and total time fell significantly on placebo compared with the 0900 exercise test on active treatment, but were not significantly different to the baseline exercise test either.

Conclusions-Intermittent transdermal treatment with glyceryl trinitrate is not associated with the rebound phenomenon in patients maintained on $\beta$ blockers for stable angina pectoris.

Royal Brompton

National Heart and

Lung Hospital,

London

D R Holdright

R J Katz

C A Wright

J L Sparrow

A K Sullivan

A D Cunningham

K M Fox

Correspondence to:

Dr Diana R Holdright,

Royal Brompton Nationa

Heart and Lung Hospital,

Sydney Street,

London SW3 6NP.

Accepted for publication

21 September 1992

\section{(Br Heart f 1993; 69:223-227)}

Organic nitrates have been used effectively in the treatment of angina pectoris for over one hundred years. ${ }^{1}$ Their short duration of action and rapid metabolism led to the development of slow release formulations and in 1982 a transdermal glyceryl trinitrate preparation was launched that provided continuous delivery of nitrate with constant plasma concentrations. ${ }^{2}$ A problem of tolerance with continuous treatment was soon recognised, however, with loss Although the mechanism of tolerance is not of effect apparent within 24 hours. ${ }^{3-6}$ well understood it may be circumvented by implementing a nitrate free interval. ${ }^{7-10}$ There is, however, evidence that intermittent regimes may predispose to anginal symptoms during the nitrate free interval, ${ }^{11-13}$ the so called rebound phenomenon, possibly due to unopposed counter regulatory mechanisms. In these studies a significant number of patients were maintained on nitrates as the only treatment, which might have rendered them more susceptible to rebound during the nitrate free interval. Not uncommonly in clinical practice patients are maintained on a combination of treatments with nitrates and a $\beta$ blocker, and potentially the problem of rebound may be lessened by this regime. This study was performed to look for evidence of rebound with serial exercise testing during nitrate withdrawal in patients also taking a $\beta$ blocker for stable angina pectoris.

\section{Patients and methods}

PATIENT SELECTION

Patients with stable angina who had had at least one positive treadmill exercise test $(\geqslant 1$ $\mathrm{mm}$ ST segment depression $80 \mathrm{~ms}$ after the J point) with associated chest pain were entered into the study. All patients were maintained on treatment with only a $\beta$ blocker throughout the trial, together with prophylactic glyceryl trinitrate (sublingual tablets or spray). Angiotensin converting enzyme inhibitors, diuretics, and aspirin were continued in 1,3 , and 6 patients respectively.

Exclusion criteria were women of child bearing age, myocardial infarction, or coronary bypass surgery within three months, unstable angina, nocturnal angina, exercise tolerance of $<3$ minutes on the modified Bruce protocol, glaucoma, relative hypotension (systolic blood pressure $<100 \mathrm{~mm} \mathrm{Hg}$ ), or other contraindication to nitrate treatment, uncontrolled hypertension, overt heart failure, inadequate control on single treatment with a $\beta$ blocker, significant arrhythmias, or baseline electrocardiographic abnormalities that precluded interpretation of ST segment changes during exercise. All patients gave written informed consent in accordance with the guidelines established by the ethics committee of our hospital.

\section{PATIENT CHARACTERISTICS}

Table 1 shows the demographic data of fourteen male patients, age $60.9(2.3)$ years (mean (SEM)). Mean duration of angina was 39.6 (range 3-108) months. Eleven patients 
Table 1 Characteristics of 14 male patients

\begin{tabular}{lc}
\hline Age (yr, mean (SEM) & $60 \cdot 9(2 \cdot 3)$ \\
Angina duration (months, mean (range)) & $39 \cdot 6(3-108)$ \\
Smoking history & $9 / 14$ \\
Hypertension & $7 / 14$ \\
Diabetes mellitus & $1 / 14$ \\
Serum cholesterol (mmol/l) & $6 \cdot 83(0 \cdot 29)$ \\
Serum triglyceride (mmol/) & $1 \cdot 77(0 \cdot 27)$ \\
Serum HDL cholesterol (mmol/l) & $1 \cdot 09(0 \cdot 07)$ \\
Previous myocardial infarction & $5 / 14$ \\
Previous coronary bypass surgery & $4 / 14$ \\
\hline
\end{tabular}

HDL, high density lipoprotein

had undergone coronary arteriography which showed significant coronary artery disease in all cases. Five patients had a history of myocardial infarction and four patients had undergone coronary artery bypass surgery. Nine patients had a smoking history (one current), seven had well controlled hypertension, and one was a non-insulin dependent diabetic.

\section{STUDY DESIGN}

The study was a randomised, double blind, placebo controlled study of cross over design, with all patients completing both treatment and placebo phases in random order (fig 1). Patients attended on three occasions and performed two symptom limited treadmill exercise tests at each visit at 0900 and 1100 . On the first visit (baseline) patients underwent treadmill testing at 0900 and 1100 to assess reproducibility. Patients were then randomised to one or other arm of the trial. At 2200 on the evening before the second visit (treatment day or withdrawal day) all patients applied one $50 \mathrm{mg}$ and one $25 \mathrm{mg}$ transdermal nitrate patch (Ciba) releasing $10 \mathrm{mg} / 24 \mathrm{~h}$ and $5 \mathrm{mg} / 24 \mathrm{hr}$ glyceryl trinitrate. If side effects (notably headache not responding to simple analgesics) warranted dosage reduction patients were instructed to remove the smaller patch. The next morning patients underwent symptom limited treadmill exercise testing at 0900 . On recovery the patch was replaced with either an active patch of equal strength or matching placebo. Patients and the supervising physician were blinded to the treatment. Two hours later a second treadmill exercise test was performed, after which the patches were removed. The schedule was identical for the third visit (withdrawal day or treatment day), with application of a nitrate patch at 2200 the previous evening.

Figure 1 Flow diagram to illustrate the study design.

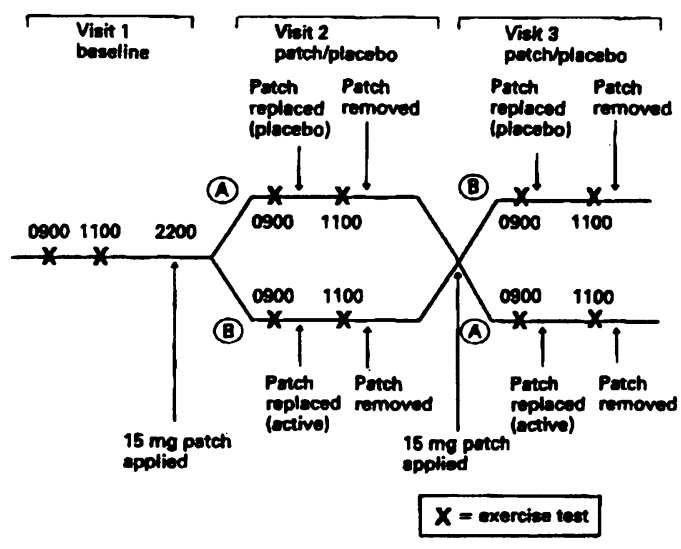

After the first exercise test on the next morning the patches were removed and replaced with either an active or a placebo patch. We looked for evidence of a rebound by comparing treadmill performance at 1100 on placebo with the 1100 exercise test at the baseline $\underline{\underline{T}}$ visit. All patients tompleted both phases, dur- $I$ ing which they received both active and placebo patches after the 0900 exercise test.

Patches were applied to a clean, dry, hairless area of the skin on the chest, avoiding $\stackrel{0}{+}$ areas of skin abrasion. The site was changed for subsequent patch application. Patients were asked about anginal symptoms and the presence of headache during the study period. The mean number of days between is visits 1 and 2 was 6.6 (range 2-14) days and $\vec{O}$ between visits 2 and 3 was $7 \cdot 5$ (range 1-15) days.

During the study all patients remained on a $\beta$ blocker, which was not stopped on the study days. Glyceryl trinitrate tablets or spray $\stackrel{\omega}{\omega}$ were not permitted for at least one hour before exercise testing. Patients were asked $\omega$

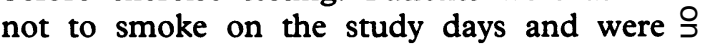
instructed to take a light early breakfast not $\vec{\overrightarrow{ }}$ less than two hours before the first exercise test. Beverages containing caffeine were not $\bar{\partial}$ permitted after this time until after completion of both exercise tests on that day.

Treadmill testing was performed with the modified Bruce protocol. Those leads that best displayed ST segment depression by a computerised, signal averaged system (Marquette Case 15) were continuously monitored. A 12 lead electrocardiogram, heart rate, and cuff blood pressure were recorded at baseline and peak exercise, and at minute intervals. Time to angina, time to 1 F $\mathrm{mm}$ ST segment depression (measured $80 \mathrm{~ms}$ after the J point), and total time were recorded and the rate-pressure product (heart rate $x$ systolic blood pressure) at each of these $\frac{3}{\exists}$ times was found. Exercise was stopped at the level of discomfort at which the patient would $\frac{0}{3}$ usually stop, or earlier if the attending physician deemed it necessary.

\section{STATISTICAL ANALYSIS}

After preliminary analysis of variance, resting $\%$ and exercise haemodynamic variables and $\tilde{O}$ time to angina, $1 \mathrm{~mm}$ ST segment depres- $\mathbb{\omega}^{-}$ sion, and total exercise time were compared ${ }_{2}$ with the paired Student's $t$ tests where appro- 0 priate. When certain end points-namely, $\mathbb{D}$ time to angina and time to $1 \mathrm{~mm} \mathrm{ST}$ segment depression-were not reached total exercise $\frac{0}{0}$ time was substituted. A p value $<0.05$ was $\underset{\mathbb{D}}{\stackrel{D}{ }}$ assumed to be significant. No significant order effect was found by crossover analysis.

Results

SIDE EFFECTS OF TREATMENT WITH GLYCERYL 을 TRINITRATE

Nine patients experienced headache while wearing the patches before the second visit and six patients experienced headache before the third visit. Most patients were controlled by simple analgesia but one patient removed 
the smaller $25 \mathrm{mg}$ patch before returning to hospital. Resting systolic blood pressure tended to be lower when on active treatment, but no compensatory tachycardia was noted, presumably due to concomitant $\beta$ blockade. There were no significant adverse events and all patients completed the study.

\section{BASELINE}

Resting haemodynamics were similar for both exercise tests, and all patients were adequately $\beta$ blocked (mean resting heart rate $<60$ beats $/ \mathrm{min}$ ). Exercise haemodynamics at time to angina, time to $1 \mathrm{~mm}$ ST segment depression, and total time were similar in both 0900 and 1100 exercise tests. Time to angina, time to $1 \mathrm{~mm} S T$ depression, and total time were not significantly different $(338 \cdot 1(50 \cdot 1)$ mean (SEM) $v 349 \cdot 1(48 \cdot 1) \mathrm{s}$ $(\mathrm{p}=0.52), 403.1(59.7)$ v 407.4 (62.2) $\mathrm{s}$ $(\mathrm{p}=0.84), 520.7(53.9)$ v $508.9(59.3) \mathrm{s}$ $(p=0.46)$, respectively). There was no evidence of a training effect.

\section{TREATMENT DAY}

On the treatment day active treatment was given during both 0900 and 1100 exercise tests. Resting heart rate and rate-pressure product were similar before both exercise
Figure 2 Bar charts to show time to angina $(A)$, time to $1 \mathrm{~mm} S T$ segment depression (B), and total exercise duration $(C)$ during the study.

$(A) * p<0.005 v$ baseline 0900 and withdrawal day $1100 ;+p<0.0005 v$ baseline 1100; $p<0.001 v$ treatment day 0900 and $1100 ;++p=0.0005 v$ treatment day 0900 and 1100.

(B) ${ }^{*} p<0.05$ v treatment day 1100 and withdrawal day 0900; $+p<0.05 v$ treatment day 0900 and 1100 and withdrawal day 0900.

$(C) \star p<0.01 v$ with drawal day 0900 and treatment day $1100 ;+p$
$<0.05$ v treatment day $1100 ;++p<0.01 v$ treatment day 1100.
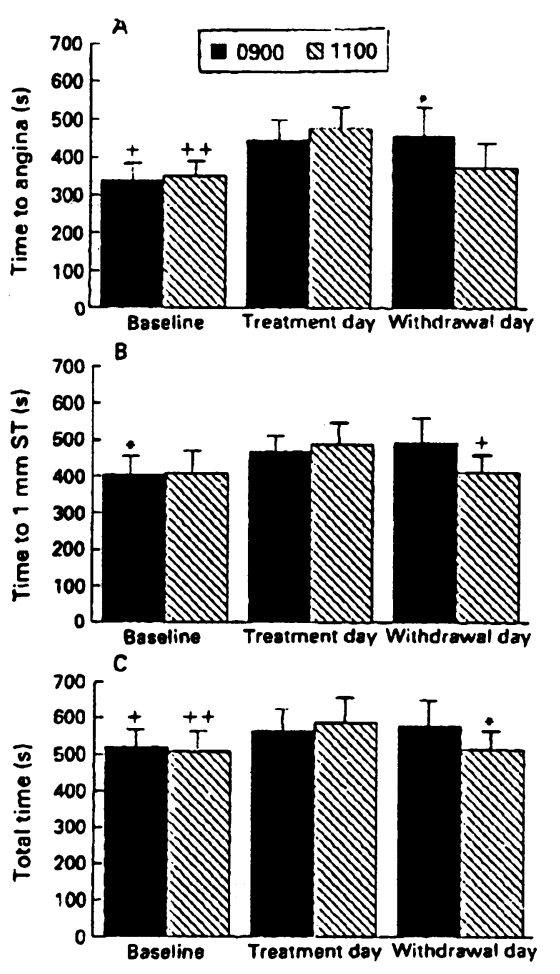

Table 2 Rate-pressure product at rest and at $1 \mathrm{~mm} S T$ segment depression during exercise testing

\begin{tabular}{|c|c|c|c|}
\hline & \multicolumn{3}{|c|}{ 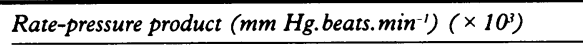 } \\
\hline & Time & Rest & $1 \mathrm{~mm} S T$ depression \\
\hline Baseline & 0900 & $8.0(0.4)$ & $13.0(0.8)$ \\
\hline \multirow{2}{*}{ Treatment day } & 1100 & $7 \cdot 6(0 \cdot 3)$ & $12.2(0.5)$ \\
\hline & 0900 & $7 \cdot 4(0 \cdot 7)$ & $12 \cdot 3(0.8)$ \\
\hline \multirow{2}{*}{ Withdrawal day } & 0900 & $\begin{array}{l}.2(0.6) \\
7.2(0.6)\end{array}$ & $12.0(0.0)$ \\
\hline & 1100 & $7 \cdot 3(0.5)$ & $11.8(0.8)$ \\
\hline
\end{tabular}

All results not significant. tests, but systolic blood pressure was significantly lower compared with the equivalent resting phase at baseline (0900 test: $125 \cdot 1$ (8.3) $v 139.6(6.2) \mathrm{mm} \mathrm{Hg}(\mathrm{p}=0.009) ; 1100$ test: $116 \cdot 1(5 \cdot 8) v 131.6(4.9) \mathrm{mm} \mathrm{Hg}(\mathrm{p}=$ 0.012 ). Similar rate-pressure products were achieved during both exercise tests (Fig 2, table 2). Time to angina, time to $1 \mathrm{~mm} \mathrm{ST}$ depression, and total time were not significantly different between the 0900 and 1100 tests on the treatment day (time to angina was $445.5(59.0) v 478.3(63.3) \mathrm{s}(\mathrm{p}=0.12)$, time to $1 \mathrm{~mm}$ ST segments depression was $466.8(56.0) v 486.7(56.8) \mathrm{s}(\mathrm{p}=0.36)$, and total time was $565 \cdot 1(57 \cdot 0) v 588.4(59 \cdot 0) \mathrm{s}$ $(p=0.44)$. Compared with the time matched baseline tests, time to angina increased significantly on the 0900 test $(p=0.0008)$, as did time to angina $(p=0.0001)$ and total time $(p=0.008)$ on the 1100 test.

\section{WITHDRAWAL DAY}

On the withdrawal day placebo treatment was given after the 0900 test. Resting systolic blood pressure was significantly lower before the 0900 test compared with the 1100 test (p $=0.046$ ) but resting heart rate and rate-pressure product were similar. Heart rate, systolic blood pressure, and rate-pressure product were similar at time to angina, at time to 1 mm ST segment depression, and at total time. Whereas four patients did not develop significant ST segment depression on the 0900 test, significant ST segment depression developed in all patients on placebo at 1100 . Similarly, angina on exercise was absent in three patients on the 09:00 test, and absent in only one patient on placebo at 1100 . Time to angina, time to $1 \mathrm{~mm}$ ST segment depression, and total time fell significantly on placebo compared with the 0900 exercise test (time to angina was $373.2(51.1) v 458.0$ $(65 \cdot 1) \mathrm{s}(\mathrm{p}=0.009)$, time to $1 \mathrm{~mm} \mathrm{ST}$ segment depression was $412.1(53.8) v 493.3$ $(66.2) \mathrm{s}(\mathrm{p}=0.02)$, and total time was 514.5 (53.1) v 578.3 (57.4) s $(\mathrm{p}=0.006))$, but were not significantly different to either the 0900 or 1100 baseline exercise tests.

\section{Discussion}

In this study we were unable to detect clinically significant rebound when the glyceryl trinitrate patch was removed after 11 hours of continuous treatment and replaced with a placebo patch in patients with background $\beta$ blockade. Subsequent exercise testing at two hours did not show any significant differences when compared with the time matched exercise test at the baseline visit when patients had not been exposed to recent treatment with glyceryl trinitrate.

Rebound occurs when adaptive responses or tolerance to the pharmacological effects of a drug are suddenly unopposed upon abrupt withdrawal of the drug. Tolerance to nitrates may involve depletion of sulphydryl donors that are necessary for converting organic nitrates to active S-nitrosothiols, ${ }^{14}$ neurohumoral activation, ${ }^{15}$ or shifts in plasma 
volume. ${ }^{16}$ Because tolerance develops so rapidly with continuous nitrate treatment, rebound is a theoretical possibility. The phenomenon of nitrate tolerance is well established and occurs rapidly with continuous treatment, ${ }^{3-6}$ regardless of the mode of delivery. The development of the nitrate patch in the early 1980s highlighted the problem. ${ }^{2}$ This transdermal delivery system was specifically designed to release nitroglycerin at a steady rate over a 24 hour period, thereby avoiding first pass inactivation by the liver and resulting in constant plasma concentrations. Although theoretically attractive, studies of delivery systems of transdermal nitrate showed that tolerance to the effect develops within 24 hours or earlier after topical application. ${ }^{3-7}$

Strategies were developed to prevent tolerance, and nitrate free intervals are generally recommended. A nitrate free interval of 10 to 12 hours seems to be adequate and patients are usually advised to remove the patch during the night. ${ }^{8-1017}$ There is, however, concern that such a regime might cause a rebound effect during the nitrate free interval, especially with the transdermal delivery system where plasma concentrations decline rapidly upon removal of the patch. ${ }^{2}$ By contrast oral nitrates, particularly the slow release formulations, have a smoother pharmacokinetic profile that theoretically would be less likely to cause rebound.

There is evidence from munitions workers exposed to glyceryl trinitrate that subsequent withdrawal may cause symptoms. Lange et al described nine patients (from a group of 200 workers in a munitions factory) who showed non-atheromatous ischaemic heart disease induced by chronic industrial exposure to glyceryl trinitrate and subsequent withdrawal. ${ }^{18}$ Reeves et al showed that in rabbits made tolerant to nitrates abrupt withdrawal of nitrates was associated with profound myocardial ischaemia, arrhythmogenesis, and death. ${ }^{19}$ Intravenous ergonovine given before nitrate treatment did not provoke myocardial ischaemia, but, after nitrate tolerance was induced, repeat ergonovine caused significant myocardial ischaemia and ventricular arrhythmias. All seven rabbits died. Muller et al describes a patient with severe Prinzmetal angina who ultimately stabilised on nifedipine and nitrates..$^{20}$ One month later nitrates were withdrawn, and 12 hours later the patient sustained a fatal myocardial infarction.

A recent report by the Transderm-Nitro Trial Study Group, which evaluated different dose schedules of nitrate patches prescribed with a 12 hour nitrate free interval in 206 patients, showed a paradoxical reduction in exercise tolerance in the treatment groups compared with the placebo arm just before patch application during long-term treatment. ${ }^{11}$ The authors postulated that this socalled "zero hour" phenomenon might be due to a rebound effect. In a study of continuous and intermittent nitrate patch regimes in stable angina, two out of 36 patients sustained a coronary event during the washout period after active treatment (one myocardial infarction, one unstable angina). ${ }^{12}$ These events may be coincidental, but rebound cannot be ruled out. After $\beta$ blocker withdrawal Ferratini et al showed an increase in spontaneous anginal attacks during the nitrate free interval (11 attacks in 10 patients) whereas no attacks occurred during continuous treatment. ${ }^{13}$ The attacks occurred at an average of 128 minutes after removal of the patch, at a time when serum nitrate concentrations decrease sharply. ${ }^{2}$ Other studies, however, suggest that anginal symptoms and ambulatory ischaemia are not exacerbated during the nitrate free interval..$^{81012122}$ These discrepancies may relate to the variability between कै studies with regard to the continuation or $\overrightarrow{0}$ withdrawal of concomitant medication. Our patients had adequate $\beta$ blockade, in that they did not develop a reflex tachycardia with the hypotensive effects of patch application and there was no significant rise in systolic blood pressure with exercise.

\section{LIMITATIONS}

We did not include an additional visit with patients maintained on placebo patches before both exercise tests to exclude possible confounding effects of the placebo itself. All patients were maintained, however, on background antianginal treatment with a $\beta$ blocker that should minimise any placebo effect from the application patch. We also recognise the possibility of a type II error with the number of patients studied. The dose or duration of nitrate treatment may have been suboptimal. One patient had to remove the $25 \mathrm{mg}$ patch due to unacceptable headache, and so underwent treadmill testing wearing the $50 \mathrm{mg}$ patch. We did not perform a dose titration when initiating nitrate treatment, but there was a significant fall in resting systolic blood pressure before exercise testing in all three active phases, compared with the time controlled baseline test, suggesting that the nitrate dose was effective. We sought evidence of rebound at two hours after removal of the patch, so conceivably rebound could have occurred at an earlier or later time. The two hour assessment time was based on the known pharmacokinetic profile of the transdermal delivery system. All patients were studied with an overnight treatment period and a morning withdrawal period. Although different results might have been seen with an inverted schedule (day time treatment and evening withdrawal) the known circadian variation of angina would have predicted a greater chance of rebound in our study.

We have been unable to show rebound after withdrawal of a glyceryl trinitrate patch in patients maintained on $\beta$ blocking agents. In those trials in which the rebound phenomenon has been observed, however, attempts were made to withdraw all other antianginal drugs. As treatment with nitrates as sole therapy is not unusual, physicians should ensure that sufficient antianginal cover with additional agents is present during the nitrate free interval to reduce the likelihood of rebound. 
As the nitrate free interval is generally nocturnal, standard prescribing regimes may need to be altered to provide adequate cover, particularly during the early morning increase in ischaemia.

1 Brunton TL. Use of nitrite in angina pectoris. Lance 1867; ii:97-8.

2 Muller P, Imhof PR, Burkart F, Chu L-C, Gerardini A Human pharmacological studies of a new transdermal system containing nitroglycerin. Eur $\mathcal{f}$ Clin Pharmacol $1982 ; 22: 473-80$

3 Crean PA, Ribeiro P, Crea F, Davies GL, Ratcliffe D, Maseri A. Failure of transdermal nitroglycerin to Maseri A. Failure improve chronic stable angina: a randomised, placebocontrolled, double-blind, double cross-over trial. $A m$

4 Parker JO, Fung H-L. Transdermal nitroglycerin in angina pectoris. Am $\mathcal{f}$ Cardiol 1984; 54:471-6.

5 Sullivan M, Savvides M, Abouantoun S, Madsen EB Froelicher V. Failure of transdermal nitroglycerin to improve exercise capacity in patients-with angina pectoris. F Am Coll Cardiol 1985; 5:1220-3.

6 Thadani U, Hamilton SF, Olson E, et al. Transdermal nitroglycerin patches in angina pectoris. Ann Int Med 1986; 105:485-92.

7 Colditz GA, Halvorsen KT, Goldhaber SZ. Randomised clinical trials of transdermal nitroglycerin systems for the treatment of angi

8 Cowan JC, Bourke JP, Reid DS, Julian DG. Prevention of tolerance to nitroglycerin patches by overnight removal. Am ₹ Cardiol 1987; 60:271-5.

9 Luke R, Sharpe N, Coxon R. Transdermal nitroglycerin in angina pectoris. Efficacy of intermittent application. $\mathcal{f}$ Am Coll Cardiol 1987; 10:642-6.

10 Schaer DH, Buff LA, Katz RJ. Sustained antianginal efficacy of transdermal nitroglycerin patches using an overnight 10-h nitrate-free interval. Am 7 Cardiol 1988 61:46-50.
11 DeMots $\mathrm{H}$, Glasser SP. Intermittent transdermal nitroglycerin therapy in the treatment of chronic stable angina. F Am Coll Cardiol 1989; 13:786-93.

12 Waters DD, Juneau M, Gossard D, Choquette G, Brian M. Limited usefulness of intermittent nitroglycerin patches in stable angina. $7 \mathrm{Am}$ Coll Cardiol 1989; 13: patches

13 Ferratini M, Pirelli S, Merlini P, Silva P, Pollavani G Intermittent transdermal nitroglycerin monotherapy in Intermittent transdermal nitroglycerin monotherapy in stable exercise-induced angina: a comparison with

14 Needleman P, Johnson EM Jr. Mechanism of tolerance development to organic nitrates. F Pharmacol Exp Ther 1987; 184:7009-15.

15 Packer $M$. What causes tolerance to nitroglycerin? The 100 year old mystery continues. 7 Am Coll Cardiol 1990; 16:932-4.

16 Dupuis J, Lalonde G, Lemieux R, Rouleau JL. Tolerance to intravenous nitroglycerin in patients with congestive heart failure: role of increased volume, neurohumoral activation and lack of prevention with $\mathrm{N}$-acetylcysteine. f Am Coll Cardiol 1990; 16:923-31.

17 Gumbrielle T, Freedman SB, Fogarty L, Ogasawara S; Sobb P, Kelly DT. Efficacy, safety and duration of nitrate-free interval to prevent tolerance to transdermal nitroglycerin in effort angina. Eur Heart $\mathcal{f} 1992 ; 13$ 671-8.

18 Lange RL, Reid MS, Tresch DD, Keelan MH, Bernhard VM, Coolidge G. Non-atheromatous ischaemic heart disease following withdrawal from chronic industria nitroglycerin exposure. Circulation 1972; 426:666-78.

19 Reeves WC, Cook L, Wood MA, Whitesall L. Coronary artery spasm after abrupt withdrawal of nitroglycerin in rabbits. Am $\mathcal{\text { f Cardiol } 1 9 8 5 ; 5 5 : 1 0 6 6 - 6 9 .}$

20 Muller JE, Gunther SJ. Nifedipine therapy for Prinzmetal's angina. Circulation 1977; 57:137-9.

21 de Milliano PA, Koster RW, Bar FW, et al Long-term efficacy of continuous and intermittent use of transdermal nitroglycerin in stable angina pectoris. $A m \mathcal{F}$ Cardiol $1991 ; 68: 857-62$.

22 Fox KM, Dargie HJ, Deanfield J, Maseri A. Avoidance of tolerance and lack of rebound with intermittent dose titrated transdermal glyceryl trinitrate. Br Heart $\mathcal{F} 1991$; 66:151-5. 